\title{
Preface
}

\section{Articular Cartilage: The Search for the Holy Grail of Treatment and Restoration}

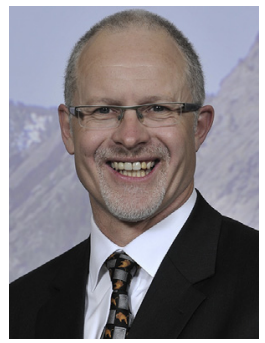

Eric C. McCarty, MD Editor

It is with great pleasure that I introduce this issue of Clinics in Sports Medicine focused on the topic of Articular Cartilage. More than ever the subject of Articular Cartilage is drawing interest in sports medicine physicians, researchers, and the public. It is a hot topic, with the treatment and science constantly evolving as we try to treat a structure that does not heal on its own after injury.

The topic of articular cartilage is an ever-changing one, and the science on articular cartilage is constantly being updated. This issue serves as an excellent update and resource for the current state-of-the-science and treatment options for articular cartilage wear in 2017.

The subject of articular cartilage conjures some type of sentiment for every sports medicine practitioner. Cartilage means something to almost everyone involved in sports medicine, whether as a competitive athlete or recreational sports enthusiast, or a practitioner, or a researcher. Every sports medicine practitioner has had a patient that has been affected by an injury to the articular cartilage, and the term "articular cartilage injury" or "damage" invokes a different image for everyone. There is such a wide spectrum that articular cartilage injury encompasses: from minimal fibrillations of superficial cartilage wear to small cartilage defects to large defects, and finally, to full-blown and widespread grade IV changes of generalized osteoarthritis. Not only have we all had patients that have been affected with a cartilage issue but also many of us have been affected either directly by incurring a cartilage injury or indirectly as a family member has been affected.

As wide as the spectrum of articular cartilage disease is, the treatment of the cartilage lesions might be even wider. The treatment can be challenging as there is a wide continuum of lesions and many options for treatment from injections to arthroplasty with no gold standard for the treatment. In addition, there are so many factors that 
affect the treatment, including but not limited to comorbidities, alignment, stability, biologic factors, patient expectations, activity level, previous surgeries, cost of procedures, and insurance issues.

The interest among the public is at an all-time high. Information on the Internet is expansive, both good and bad. With so much of the public affected, the interest in treatment options, particularly nonsurgical options, has significantly risen and so have the unproven treatments and the public's willingness to pay large amounts of cash in hope of finding the "Holy Grail" treatment. Much is needed in science and studies to understand the nonsurgical options.

A recent concept introduced by Dr Fred Nelson is the idea that a joint should be considered an organ. As one uses this perspective, then any cartilage injury is not treated in isolation. There is a milieu of issues that can contribute to the health of articular cartilage, including mechanical alignment of the joint, stability, and factors that increase the mechanical pressures in the joint (ie, removal of meniscus tissue in the knee). It is beyond the scope of this issue to go into all of the issues associated with the health of the joints and factors that contribute to articular cartilage injury and health, but suffice it to say, this issue of Clinics in Sports Medicine will delve into the salient factors involved in the science of articular cartilage, the imaging, the physiology of early osteoarthritis, and the myriad of nonoperative and operative options of articular cartilage restoration for the most affected joints in the body (knee, hip, shoulder).

A big thanks to Mark Miller for allowing me the opportunity to corral and bring together my colleagues and leaders of sports medicine for this issue, and, to my sweet wife, Miriam, who puts up with long hours and often late nights so that the "calling" of the academic mission of efforts such as this can be fulfilled.

I applaud the renowned experts and leaders in the ever-changing field of articular cartilage that have worked hard and made terrific thoughtful contributions to this issue. As you read this issue of Clinics in Sports Medicine, enjoy the content, the information, and ideas that have been presented. These articles represent the latest information for the practitioner on the topic of articular cartilage. There is something for everyone: for the new student just trying to understand the concepts to the gray-haired sports medicine physician with many years of experience trying to garner new information. As you read, also understand that this is an evolving subject and that information is constantly being renewed and evaluated. It will be very interesting to see what the Clinics in Sports Medicine issue of articular cartilage will look like two decades from now. I would expect that many of the articles will be obsolete, and there will be many more articles on the biological treatment of cartilage disease with an emphasis on disease-modifying modalities. Enjoy this issue. It is my hope that the contents spur thoughts and ideas that will help shape the research and techniques that contribute to the articular cartilage issue of Clinics in Sports Medicine in the year 2027.

Many blessings to you as you learn more in these readings and in your pursuit for the Holy Grail in the treatment of articular cartilage injury.

Eric C. McCarty, MD University of Colorado

CU Sports Medicine

Champions Center

2150 Stadium Drive Boulder, CO 80309, USA 